\title{
On Real Matrices to Least-Squares g-Inverse and Minimum Norm g-Inverse of Quaternion Matrices*
}

\author{
Huasheng Zhang \\ School of Mathematics Science, Liaocheng University, Shandong, China \\ Email: zhsh0510@yahoo.com.cn
}

Received November 20, 2011; revised December 24, 2011; accepted December 30, 2011

\begin{abstract}
Through the real representations of quaternion matrices and matrix rank method, we give the expression of the real matrices in least-squares g-inverse and minimum norm g-inverse. From these formulas, we derive the extreme ranks of the real matrices. As applications, we establish necessary and sufficient conditions for some special least-squares g-inverse and minimum norm g-inverse.
\end{abstract}

Keywords: Extreme Rank; g-Inverse; Least-Squares g-Inverse; Minimum Norm g-Inverse; Quaternion Matrix

\section{Introduction}

Throughout this paper, $\mathbb{R}$ stands for the real number field, $\mathbb{H}^{m \times n}$ stands for the set of all $m \times n$ matrices over the quaternion algebra

$$
\begin{aligned}
\mathbb{H}=\{ & a_{0}+a_{1} i+a_{2} j+a_{3} k \mid \dot{i}^{2}=j^{2}=k^{2}=i j k=-1, \\
& \left.a_{0}, a_{1}, a_{2}, a_{3} \in \mathbb{R}\right\} .
\end{aligned}
$$

$I, A^{T}, A^{*}$ and $A^{\dagger}$ stand for the identity matrix' the transpose' the conjugate transpose and the Moore-Penrose inverse of a quaternion matrix $A$. In [1], for a quaternion matrix $A, \operatorname{dim} \mathcal{R}(A)=\operatorname{dim} \mathcal{N}(A) . \quad \operatorname{dim} \mathcal{R}(A)$ is called the rank of a quaternion matrix $A$ and denoted by $r(A)$.

The well known Moore-Penrose inverse $A^{\dagger}$ of $A \in \mathbb{H}^{m \times n}$ is defined to be the unique matrix $X \in \mathbb{H}^{m \times n}$ satisfying the following four Penrose equations

1) $A X A=A$,

2) $X A X=X$,

3) $(A X)^{*}=A X$

4) $(X A)^{*}=X A$.

A matrix $X$ is called a least-squares g-inverse of $A$ if it satisfies both 1) and 3 ) in the Penrose equations, and denoted by $A^{(1,3)}$; a matrix $X$ is called a minimum norm g-inverse of $A$ if it satisfies both 1) and 4) in the Penrose equations, and denoted by $A^{(1,4)}$. The general expression of $A^{(1,3)}$ and $A^{(1,4)}$ can be written as

$$
\begin{aligned}
& A^{(1,3)}=A^{\dagger}+L_{A} V, \\
& A^{(1,4)}=A^{\dagger}+W R_{A}
\end{aligned}
$$

*This research was supported by the Natural Science Foundation of China (11001115). where $L_{\mathrm{A}}=I-A^{\dagger} A, \quad R_{\mathrm{A}}=I-A A^{\dagger}$, the two matrices $V$ and $W$ is a arbitrary; see [[2], pp. 44-46].

For convenience of representation, we suppose

$$
A=A_{0}+A_{1} i+A_{2} j+A_{3} k
$$

and

$$
\begin{aligned}
& A^{(1,3)}=B_{0}+B_{1} i+B_{2} j+B_{3} k, \\
& A^{(1,4)}=C_{0}+C_{1} i+C_{2} j+C_{3} k
\end{aligned}
$$

where $A_{0}, A_{1}, A_{2}, A_{3} \in \mathbb{R}^{m \times n}, \quad B_{0}, B_{1}, B_{2}, B_{3} \in \mathbb{R}^{m \times n}$,

$$
C_{0}, C_{1}, C_{2}, C_{3} \in \mathbb{R}^{n \times m} .
$$

For an arbitrary quaternion matrix

$$
M=M_{1}+M_{2} i+M_{3} j+M_{4} k,
$$

we define a map $\phi(\cdot)$ from $\mathbb{H}^{m \times n}$ to $\mathbb{R}^{4 m \times 4 n}$ by

$$
\phi(M)=\left[\begin{array}{cccc}
M_{4} & M_{3} & M_{2} & M_{1} \\
-M_{3} & M_{4} & M_{1} & -M_{2} \\
M_{2} & M_{1} & -M_{4} & -M_{3} \\
M_{1} & -M_{2} & M_{3} & -M_{4}
\end{array}\right] .
$$

By (5), it is easy to verify that $\phi(\cdot)$ satisfies the following properties:

a) $M=N \Leftrightarrow \phi(M)=\phi(N)$.

b) $\phi(M+N)=\phi(M)+\phi(N), \phi(M N)=\phi(M) \phi(N)$,

$$
\phi(k M)=k \phi(M), k \in \mathbb{R} .
$$

c) $\phi(M)=-T_{m}^{-1} \phi(M) T_{n}=-R_{m}^{-1} \phi(M) R_{n}$

$$
=S_{m}^{-1} \phi(M) S_{n},
$$

where 


$$
\begin{aligned}
& T_{t}=\left[\begin{array}{cccc}
0 & -I_{t} & 0 & 0 \\
I_{t} & 0 & 0 & 0 \\
0 & 0 & 0 & I_{t} \\
0 & 0 & -I_{t} & 0
\end{array}\right], R_{t}=\left[\begin{array}{cccc}
0 & 0 & -I_{t} & 0 \\
0 & 0 & 0 & -I_{t} \\
I_{t} & 0 & 0 & 0 \\
0 & I_{t} & 0 & 0
\end{array}\right], \\
& S_{t}=\left[\begin{array}{cccc}
0 & 0 & 0 & -I_{t} \\
0 & 0 & I_{t} & 0 \\
0 & -I_{t} & 0 & 0 \\
I_{t} & 0 & 0 & 0
\end{array}\right], t=m, n . \\
& \text { d) } r[\phi(M)]=4 r(M) .
\end{aligned}
$$

The least-squares g-inverse and minimum norm g-inverse have a very wide range of applications in numerical analysis and mathematical statistics and have been examined by many authors(see, e.g., [3-6]). Haruo [3] developed some equivalent conditions on least-squares general inverse in 1990. Tian [4] presented the maximal and minimal ranks of the Schur complement to least-squares g-inverse and minimum norm g-inverse in 2004. Tian [5] establish necessary and sufficient conditions for a matrix to be the least-squares g-inverse and minimum norm g-inverse from rank formulas in 2005. Guo, Wei and Wang [6] derived structures of least squares g-inverses and minimum norm g-inverse of a bordered matrix in 2006.

Quaternion matrices play an important role in mechanics, computer science, quantum physics, signal and color image processing and so on. More and more interests of quaternion matrices have been witnessed recently (e.g. [7-14]).

Noticing that the properties of the real matrices in least-squares general inverse $A^{(1,3)}$ and minimum norm g-inverse $A^{(1,4)}$ (4) have not been considered so far in the literature. We in this paper use the real representations of quaternion matrices and matrix rank method to investigate (4) over $\mathbb{H}$. In Section 2, we first give the expression of the real matrices $B_{i}$ and $C_{i}(i=0,1,2,3)$ in (4), then determine the maximal and minimal ranks of the real matrices $B_{i}$ and $C_{i}(i=0,1,2,3)$ in (4). As applications, we establish necessary and sufficient conditions for a quaternion matrix has a pure real or pure imaginary $A^{(1,3)}$ and $A^{(1,4)}$. The necessary and sufficient conditions for all $A^{(1,3)}$ and $A^{(1,4)}$ are pure real or pure imaginary of a quaternion matrix are also presented.

\section{Main Results}

We begin with the following lemmas which proof just like those over the complex field.

Lemma 2.1 (see [15]) Let $A, B$ and $C$ be $m \times n, m \times k, l \times n$ matrices over $\mathbb{H}$. Then

a) $r[A, B]=r(A)+r\left(R_{A} B\right)=r(B)+r\left(R_{B} A\right)$, b) $r\left[\begin{array}{l}A \\ C\end{array}\right]=r(A)+r\left(C L_{A}\right)=r(C)+r\left(A L_{C}\right)$,

c) $r\left[\begin{array}{ll}A & B \\ C & 0\end{array}\right]=r(B)+r(C)+r\left(R_{B} A L_{C}\right)$.

Lemma 2.2 (see [16]) Let $A \in \mathbb{H}^{m \times n}, \quad B \in \mathbb{H}^{m \times p}$, $C \in \mathbb{H}^{q \times n}$ be given. Then

a) The maximal rank of $A-B X C$ with respect to $X$ is

$$
\max _{X} r(A-B X C)=\min \left\{r\left[\begin{array}{ll}
A & B
\end{array}\right], r\left[\begin{array}{l}
A \\
C
\end{array}\right]\right\} ;
$$

b) The minimal rank of $A-B X C$ with respect to $X$ is

$$
\min _{X} r(A-B X C)=r\left[\begin{array}{ll}
A & B
\end{array}\right]+r\left[\begin{array}{l}
A \\
C
\end{array}\right]-r\left[\begin{array}{ll}
A & B \\
C & 0
\end{array}\right] .
$$

Theorem 2.3 Suppose $\left[X_{i j}\right]_{4 \times 4}(i, j=1,2,3,4)$ is a leastsquares g-inverse of $\phi(A)$, where $X_{i j} \in \mathbb{R}^{n \times m}, A$ and $A^{(1,3)}$ are defined as (3) and (4). Then $B_{i}(i=0,1,2,3)$ in (4) can be written as

$$
\begin{aligned}
& B_{0}=\frac{1}{4}\left(X_{11}+X_{22}-X_{33}-X_{44}\right), \\
& B_{1}=\frac{1}{4}\left(X_{12}-X_{21}+X_{43}-X_{34}\right), \\
& B_{2}=\frac{1}{4}\left(X_{13}+X_{31}-X_{24}-X_{42}\right), \\
& B_{3}=\frac{1}{4}\left(X_{41}+X_{14}+X_{32}+X_{23}\right),
\end{aligned}
$$

Written in an explicit form, $B_{i}(i=0,1,2,3)$ in (4) are

$$
\begin{aligned}
B_{0}= & \frac{1}{4} P_{1} \phi\left(A^{\dagger}\right) Q_{1}+\frac{1}{4} P_{2} \phi\left(A^{\dagger}\right) Q_{2} \\
& -\frac{1}{4} P_{3} \phi\left(A^{\dagger}\right) Q_{3}-\frac{1}{4} P_{4} \phi\left(A^{\dagger}\right) Q_{4} \\
& +\left[P_{1} L_{\phi(A)}, P_{2} L_{\phi(A)}, P_{3} L_{\phi(A)}, P_{4} L_{\phi(A)}\right]\left[\begin{array}{c}
V_{1} \\
V_{2} \\
V_{3} \\
V_{4}
\end{array}\right], \\
B_{1}= & \frac{1}{4} P_{1} \phi\left(A^{\dagger}\right) Q_{2}-\frac{1}{4} P_{2} \phi\left(A^{\dagger}\right) Q_{1} \\
& +\frac{1}{4} P_{4} \phi\left(A^{\dagger}\right) Q_{3}-\frac{1}{4} P_{3} \phi\left(A^{\dagger}\right) Q_{4} \\
& +\left[P_{1} L_{\phi(A)}, P_{2} L_{\phi(A)}, P_{4} L_{\phi(A)}, P_{3} L_{\phi(A)}\right]\left[\begin{array}{c}
V_{2} \\
-V_{1} \\
V_{3} \\
-V_{4}
\end{array}\right],
\end{aligned}
$$




$$
\begin{aligned}
B_{2}= & \frac{1}{4} P_{1} \phi\left(A^{\dagger}\right) Q_{3}-\frac{1}{4} P_{2} \phi\left(A^{\dagger}\right) Q_{4} \\
& +\frac{1}{4} P_{3} \phi\left(A^{\dagger}\right) Q_{1}-\frac{1}{4} P_{4} \phi\left(A^{\dagger}\right) Q_{2} \\
& +\left[P_{1} L_{\phi(A)}, P_{3} L_{\phi(A)},-P_{2} L_{\phi(A)},-P_{4} L_{\phi(A)}\right]\left[\begin{array}{c}
V_{3} \\
V_{1} \\
-V_{4} \\
-V_{2}
\end{array}\right],
\end{aligned}
$$$$
B_{3}=\frac{1}{4} P_{1} \phi\left(A^{\dagger}\right) Q_{4}+\frac{1}{4} P_{2} \phi\left(A^{\dagger}\right) Q_{3}
$$$$
+\frac{1}{4} P_{3} \phi\left(A^{\dagger}\right) Q_{2}+\frac{1}{4} P_{4} \phi\left(A^{\dagger}\right) Q_{1}
$$$$
+\frac{1}{4}\left[P_{1} L_{\phi(A)}, P_{4} L_{\phi(A)}, P_{3} L_{\phi(A)}, P_{2} L_{\phi(A)}\right]\left[\begin{array}{c}
V_{4} \\
V_{1} \\
V_{2} \\
V_{3}
\end{array}\right],
$$

where

$$
\begin{aligned}
& P_{1}=\left[I_{n}, 0,0,0\right], P_{2}=\left[0, I_{n}, 0,0\right], \\
& P_{3}=\left[0,0, I_{n}, 0\right], P_{4}=\left[0,0,0, I_{n}\right], \\
& Q_{1}=\left[I_{m}, 0,0,0\right]^{T}, Q_{2}=\left[0, I_{m}, 0,0\right]^{T}, \\
& Q_{3}=\left[0,0, I_{m}, 0\right]^{T}, Q_{4}=\left[0,0,0, I_{m}\right]^{T},
\end{aligned}
$$

and $V_{1}, V_{2}, V_{3}$ and $V_{4}$ are arbitrary real matrices with compatible sizes.

Proof. Suppose $\left[X_{i j}\right]_{4 \times 4}(i, j=1,2,3,4)$ is a leastsquares g-inverse of $\phi(A)$, where $X_{i j} \in \mathbb{R}^{n \times m}$, i.e.

$$
\begin{aligned}
& \phi(A)\left[X_{i j}\right]_{4 \times 4} \phi(A)=\phi(A), \\
& \left(\phi(A)\left[X_{i j}\right]_{4 \times 4}\right)^{*}=\phi(A)\left[X_{i j}\right]_{4 \times 4} .
\end{aligned}
$$

Then applying property (c) of $\phi(\cdot)$ above to them yields

$$
\begin{aligned}
& T_{m}^{-1} \phi(A) T_{n}\left[X_{i j}\right]_{4 \times 4} T_{m}^{-1} \phi(A) T_{n}=-T_{m}^{-1} \phi(A) T_{n}, \\
& \left(T_{m}^{-1} \phi(A) T_{n}\left[X_{i j}\right]_{4 \times 4}\right)^{*}=T_{m}^{-1} \phi(A) T_{n}\left[X_{i j}\right]_{4 \times 4} ; \\
& R_{m}^{-1} \phi(A) R_{n}\left[X_{i j}\right]_{4 \times 4} R_{m}^{-1} \phi(A) R_{n}=-R_{m}^{-1} \phi(A) R_{n}, \\
& \left(R_{m}^{-1} \phi(A) R_{n}\left[X_{i j}\right]_{4 \times 4}\right)^{*}=R_{m}^{-1} \phi(A) R_{n}\left[X_{i j}\right]_{4 \times 4} ; \\
& S_{m}^{-1} \phi(A) S_{n}\left[X_{i j}\right]_{4 \times 4} S_{m}^{-1} \phi(A) S_{n}=S_{m}^{-1} \phi(A) S_{n}, \\
& \left(S_{m}^{-1} \phi(A) S_{n}\left[X_{i j}\right]_{4 \times 4}\right)^{*}=S_{m}^{-1} \phi(A) S_{n}\left[X_{i j}\right]_{4 \times 4} .
\end{aligned}
$$

Hence,

$$
\begin{aligned}
& \phi(A) T_{n}\left[X_{i j}\right]_{4 \times 4} T_{m}^{-1} \phi(A)=\phi(A), \\
& \left(\phi(A) T_{n}\left[X_{i j}\right]_{4 \times 4} T_{m}^{-1}\right)^{*}=\phi(A) T_{n}\left[X_{i j}\right]_{4 \times 4} T_{m}^{-1} ;
\end{aligned}
$$

$$
\begin{aligned}
& \phi(A) R_{n}\left[X_{i j}\right]_{4 \times 4} R_{m}^{-1} \phi(A)=\phi(A), \\
& \left(\phi(A) R_{n}\left[X_{i j}\right]_{4 \times 4} R_{m}^{-1}\right)^{*}=\phi(A) R_{n}\left[X_{i j}\right]_{4 \times 4} R_{m}^{-1} ; \\
& \phi(A) S_{n}\left[X_{i j}\right]_{4 \times 4} S_{m}^{-1} \phi(A)=\phi(A), \\
& \left(\phi(A) S_{n}\left[X_{i j}\right]_{4 \times 4} S_{m}^{-1}\right)^{*}=\phi(A) S_{n}\left[X_{i j}\right]_{4 \times 4} S_{m}^{-1},
\end{aligned}
$$

which implies that $T_{n}\left[X_{i j}\right]_{4 \times 4} T_{m}^{-1}, R_{n}\left[X_{i j}\right]_{4 \times 4} R_{m}^{-1}$ and $S_{n}\left[X_{i j}\right]_{4 \times 4} S_{m}^{-1}$ are also least-squares g-inverses of $\phi(A)$. Thus,

$$
\begin{aligned}
& \frac{1}{4}\left(\left[X_{i j}\right]_{4 \times 4}-T_{n}\left[X_{i j}\right]_{4 \times 4} T_{m}^{-1}-R_{n}\left[X_{i j}\right]_{4 \times 4} R_{m}^{-1}\right. \\
& \left.\quad+S_{n}\left[X_{i j}\right]_{4 \times 4} S_{m}^{-1}\right)
\end{aligned}
$$

is also a least-squares g-inverse of $\phi(A)$, where

$$
\begin{aligned}
& {\left[X_{i j}\right]_{4 \times 4}-T_{n}\left[X_{i j}\right]_{4 \times 4} T_{m}^{-1}-R_{n}\left[X_{i j}\right]_{4 \times 4} R_{m}^{-1}} \\
& +S_{n}\left[X_{i j}\right]_{4 \times 4} S_{m}^{-1}=\left[\widehat{X}_{i j}\right]_{4 \times 4}
\end{aligned}
$$

and

$$
\begin{aligned}
& \widehat{X}_{11}=X_{11}+X_{22}-X_{33}-X_{44}, \\
& \widehat{X}_{12}=X_{12}-X_{21}+X_{43}-X_{34}, \\
& \widehat{X}_{13}=X_{13}+X_{31}-X_{24}-X_{42}, \\
& \widehat{X}_{14}=X_{41}+X_{14}+X_{32}+X_{23}, \\
& \widehat{X}_{21}=X_{21}-X_{12}+X_{34}-X_{43}, \\
& \widehat{X}_{22}=X_{11}+X_{22}-X_{33}-X_{44}, \\
& \widehat{X}_{23}=X_{41}+X_{14}+X_{32}+X_{23}, \\
& \widehat{X}_{24}=X_{24}+X_{42}-X_{13}-X_{31}, \\
& \widehat{X}_{31}=X_{13}+X_{31}-X_{24}-X_{42}, \\
& \widehat{X}_{32}=X_{41}+X_{14}+X_{32}+X_{23}, \\
& \widehat{X}_{33}=X_{33}+X_{44}-X_{11}-X_{22}, \\
& \widehat{X}_{34}=X_{21}-X_{12}+X_{34}-X_{43}, \\
& \widehat{X}_{41}=X_{41}+X_{14}+X_{32}+X_{23}, \\
& \widehat{X}_{42}=X_{24}+X_{42}-X_{13}-X_{31}, \\
& \widehat{X}_{43}=X_{12}-X_{21}+X_{43}-X_{34}, \\
& \widehat{X}_{44}=X_{33}+X_{44}-X_{11}-X_{22},
\end{aligned}
$$


Let

$$
\begin{aligned}
\widetilde{X}= & \frac{1}{4}\left(X_{11}+X_{22}-X_{33}-X_{44}\right) \\
& +\frac{1}{4}\left(X_{12}-X_{21}+X_{43}-X_{34}\right) i \\
& +\frac{1}{4}\left(X_{13}+X_{31}-X_{24}-X_{42}\right) j \\
& +\frac{1}{4}\left(X_{41}+X_{14}+X_{32}+X_{23}\right) k,
\end{aligned}
$$

Then, by (5),

$$
\begin{aligned}
\phi(\widetilde{X})=\frac{1}{4} & \left(\left[X_{i j}\right]_{4 \times 4}-T_{n}\left[X_{i j}\right]_{4 \times 4} T_{m}^{-1}\right. \\
& \left.-R_{n}\left[X_{i j}\right]_{4 \times 4} R_{m}^{-1}+S_{n}\left[X_{i j}\right]_{4 \times 4} S_{m}^{-1}\right),
\end{aligned}
$$

is a least-squares g-inverse of $\phi(A)$. Hence, by the property (a) of $\phi(\cdot)$ we know that $\hat{X}$ a least-squares g-inverse of $A$. The above discussion shows that the least-squares g-inverse of $\phi(A)$ and the least-squares g-inverse of $A$ are equivalent. Observe that $X_{i j}, i, j=$ $1,2,3,4$ in (8) can be written as

$$
X_{i j}=P_{i} \hat{X} Q_{j} .
$$

From (1), the least-squares g-inverse of $\phi(A)$ can be written as

$$
\widetilde{X}=\phi\left(A^{\dagger}\right)+L_{\phi(A)} V
$$

where $V=\left(V_{1}, V_{2}, V_{3}, V_{4}\right)$ and $V_{1}, V_{2}, V_{3}, V_{4} \in \mathbb{R}^{4 p \times q}$ are arbitrary.

Substituting them into (8) yields the four real matrices $B_{0}, B_{1}, B_{2}$ and $B_{3}$ in (9)-(12).

According to Lemma 2 and Theorem 2, we can get the following extreme ranks formulas for the real matrices in the least-squares g-inverses.

Theorem 2.4 Suppose that $A$ and $A^{(1,3)}$ are defined as (3) and (4). Then

a) $\max r\left(B_{0}\right)=\min \left\{r\left[\begin{array}{ll}\bar{A}_{0} & \tilde{A}\end{array}\right]-4 r(A)+n, m\right\}$;

$$
\min r\left(B_{0}\right)=r\left[\begin{array}{ll}
\bar{A}_{0} & \widetilde{A}
\end{array}\right]-r(\widetilde{A}) .
$$

b) $\max r\left(B_{1}\right)=\min \left\{r\left[\begin{array}{ll}\bar{A}_{1} & \tilde{A}\end{array}\right]-4 r(A)+n, m\right\}$;

$$
\min r\left(B_{1}\right)=r\left[\begin{array}{ll}
\bar{A}_{1} & \widetilde{A}
\end{array}\right]-r(\widetilde{A}) \text {. }
$$

c) $\max r\left(B_{2}\right)=\min \left\{r\left[\begin{array}{ll}\bar{A}_{2} & \widetilde{A}\end{array}\right]-4 r(A)+n, m\right\}$;

$$
\min r\left(B_{2}\right)=r\left[\begin{array}{ll}
\bar{A}_{2} & \widetilde{A}
\end{array}\right]-r(\widetilde{A}) .
$$

d) $\max r\left(B_{3}\right)=\min \left\{r\left[\begin{array}{ll}\bar{A}_{3} & \tilde{A}\end{array}\right]-4 r(A)+n, m\right\}$;

$$
\min r\left(B_{3}\right)=r\left[\begin{array}{ll}
\bar{A}_{3} & \widetilde{A}
\end{array}\right]-r(\widetilde{A}),
$$

where

$$
\begin{aligned}
& \bar{A}_{0}=\left[\begin{array}{c}
-\hat{A}_{0} \\
-\hat{A}_{1} \\
\hat{A}_{2} \\
\hat{A}_{3}
\end{array}\right], \bar{A}_{1}=\left[\begin{array}{c}
-\hat{A}_{1} \\
\hat{A}_{0} \\
-\hat{A}_{2} \\
\hat{A}_{3}
\end{array}\right], \bar{A}_{2}=\left[\begin{array}{c}
-\hat{A}_{2} \\
-\hat{A}_{0} \\
\hat{A}_{3} \\
\hat{A}_{1}
\end{array}\right], \bar{A}_{3}=\left[\begin{array}{c}
\hat{A}_{3} \\
\hat{A}_{0} \\
\hat{A}_{1} \\
\hat{A}_{2}
\end{array}\right], \\
& \widetilde{A}=\left[\begin{array}{cccc}
\widetilde{A}_{0} & 0 & 0 & 0 \\
0 & \tilde{A}_{1} & 0 & 0 \\
0 & 0 & \tilde{A}_{2} & 0 \\
0 & 0 & 0 & \widetilde{A}_{3}
\end{array}\right], \widetilde{A}_{0}=\left[\begin{array}{ccc}
-A_{1} & -A_{2} & -A_{3} \\
A_{0} & A_{3} & -A_{2} \\
-A_{3} & A_{0} & A_{1} \\
A_{2} & -A_{1} & A_{0}
\end{array}\right],
\end{aligned}
$$

and

$$
\begin{aligned}
& \hat{A}_{0}=\left[\begin{array}{l}
A_{0} \\
A_{1} \\
A_{2} \\
A_{3}
\end{array}\right], \hat{A}_{1}=\left[\begin{array}{c}
-A_{1} \\
A_{0} \\
-A_{3} \\
A_{2}
\end{array}\right], \hat{A}_{2}=\left[\begin{array}{c}
-A_{2} \\
A_{3} \\
A_{0} \\
-A_{1}
\end{array}\right], \hat{A}_{3}=\left[\begin{array}{c}
-A_{3} \\
-A_{2} \\
A_{1} \\
A_{0}
\end{array}\right], \\
& \widetilde{A}_{1}=\left[\begin{array}{ccc}
A_{0} & -A_{2} & -A_{3} \\
A_{1} & A_{3} & -A_{2} \\
A_{2} & A_{0} & A_{1} \\
A_{3} & -A_{1} & A_{0}
\end{array}\right], \widetilde{A}_{2}=\left[\begin{array}{ccc}
A_{0} & -A_{1} & -A_{3} \\
A_{1} & A_{0} & -A_{2} \\
A_{2} & -A_{3} & A_{1} \\
A_{3} & A_{2} & A_{0}
\end{array}\right], \\
& \widetilde{A}_{3}=\left[\begin{array}{ccc}
A_{0} & -A_{1} & -A_{2} \\
A_{1} & A_{0} & A_{3} \\
A_{2} & -A_{3} & A_{0} \\
A_{3} & A_{2} & -A_{1}
\end{array}\right] .
\end{aligned}
$$

Proof. Applying (6) and (7) to $B_{0}$ in (9), we get the following

$$
\begin{aligned}
& \max r\left(B_{0}\right)=\min \left\{r\left[\begin{array}{ll}
\hat{P} & \widetilde{P}
\end{array}\right], r\left[\begin{array}{c}
\hat{P} \\
I_{m}
\end{array}\right]\right\} ; \\
& \min r\left(B_{0}\right)=r\left[\begin{array}{ll}
\hat{P} & \widetilde{P}
\end{array}\right]+r[\hat{P}]-r\left[\begin{array}{cc}
\hat{P} & \widetilde{P} \\
I_{m} & 0
\end{array}\right],
\end{aligned}
$$

where

$$
\begin{aligned}
\widehat{P}= & \frac{1}{4} P_{1} \phi\left(A^{\dagger}\right) Q_{1}+\frac{1}{4} P_{2} \phi\left(A^{\dagger}\right) Q_{2} \\
& -\frac{1}{4} P_{3} \phi\left(A^{\dagger}\right) Q_{3}-\frac{1}{4} P_{4} \phi\left(A^{\dagger}\right) Q_{4}, \\
\widetilde{P}= & {\left[P_{1} L_{\phi(A)}, P_{2} L_{\phi(A)}, P_{3} L_{\phi(A)}, P_{4} L_{\phi(A)}\right] . }
\end{aligned}
$$

By Lemma 1, it is not difficult to find that 


$$
\begin{aligned}
& r[\hat{P} \quad \widetilde{P}] \\
= & r\left[\begin{array}{ccccc}
\hat{P} & P_{1} & P_{2} & P_{3} & P_{4} \\
0 & \phi(A) & 0 & 0 & 0 \\
0 & 0 & \phi(A) & 0 & 0 \\
0 & 0 & 0 & \phi(A) & 0 \\
0 & 0 & 0 & 0 & \phi(A)
\end{array}\right]-4 r[\phi(A)] \\
= & r\left[\begin{array}{ccccc}
0 & P_{1} & P_{2} & P_{3} & P_{4} \\
\hat{A}_{0} & \phi(A) & 0 & 0 & 0 \\
\hat{A}_{1} & 0 & \phi(A) & 0 & 0 \\
\hat{A}_{2} & 0 & 0 & \phi(A) & 0 \\
\hat{A}_{3} & 0 & 0 & 0 & \phi(A)
\end{array}\right]-4 r[\phi(A)] \\
= & r\left[\begin{array}{cc}
\bar{A}_{0} & \widetilde{A}]-4 r(A)+n,
\end{array}\right.
\end{aligned}
$$

where $\hat{A}_{0}, \hat{A}_{1}, \hat{A}_{2}, \hat{A}_{3}$ and $\widetilde{A}$ are defined as above. By the same manner, we can get extreme ranks of $B_{1}, B_{2}$ and $B_{3}$.

As one of important applications of the maximal and minimal ranks to real matrices, Theorem 2 can help to get the necessary and sufficient conditions for the existence of some special least-squares g-inverses. We show them in the following.

Corollary 2.5 Suppose $A=A_{0}+A_{1} i+A_{2} j+A_{3} k$.

Then

a) A quaternion matrix $A$ has a real least-squares g-inverse if and only if

$$
r\left[\begin{array}{ll}
\bar{A}_{1} & \tilde{A}
\end{array}\right]=r\left[\begin{array}{ll}
\bar{A}_{2} & \widetilde{A}
\end{array}\right]=r\left[\begin{array}{ll}
\bar{A}_{3} & \tilde{A}
\end{array}\right]=r(\widetilde{A}) .
$$

b) All least-squares g-inverses of quaternion matrix $A$ are real matrices if and only if

$$
r\left[\begin{array}{ll}
\bar{A}_{1} & \tilde{A}
\end{array}\right]=r\left[\begin{array}{ll}
\bar{A}_{2} & \tilde{A}
\end{array}\right]=r\left[\begin{array}{ll}
\bar{A}_{3} & \tilde{A}
\end{array}\right]=4 r(A)-n,
$$

where $\bar{A}_{1}, \bar{A}_{2}, \bar{A}_{3}$ and $\widetilde{A}$ are defined as Theorem 2.

Corollary 2.6 Suppose $A=A_{0}+A_{1} i+A_{2} j+A_{3} k$. Then

a) A quaternion matrix $A$ has a pure imaginary leastsquares $g$-inverse if and only if

$$
r\left[\begin{array}{ll}
\bar{A}_{0} & \widetilde{A}
\end{array}\right]=r(\widetilde{A}) .
$$

b) All least-squares g-inverses of quaternion matrix $A$ are pure imaginary matrices if and only if

$$
r\left[\begin{array}{ll}
\bar{A}_{0} & \tilde{A}
\end{array}\right]=4 r(A)-n,
$$

where $\bar{A}_{0}$ and $\widetilde{A}$ are defined as Theorem 2.

The following several theorems of minimum norm g-inverse can be shown by a similar approach, and their proofs are omitted here.

Theorem 2.7 Suppose $\left[Y_{i j}\right]_{4 \times 4}(i, j=1,2,3,4)$ is a minimum norm g-inverse of $\phi(A)$, where $Y_{i j} \in \mathbb{R}^{n \times m}$,
A and $A^{(1,4)}$ are defined as (3) and (4) Then $C_{i}(i=0,1,2,3)$ in (4) can be written as

$$
\begin{aligned}
& C_{0}=\frac{1}{4}\left(Y_{11}+Y_{22}-Y_{33}-Y_{44}\right), \\
& C_{1}=\frac{1}{4}\left(Y_{12}-Y_{21}+Y_{43}-Y_{34}\right), \\
& C_{2}=\frac{1}{4}\left(Y_{13}+Y_{31}-Y_{24}-Y_{42}\right), \\
& C_{3}=\frac{1}{4}\left(Y_{41}+Y_{14}+Y_{32}+Y_{23}\right) .
\end{aligned}
$$

Written in an explicit form, $C_{i}(i=0,1,2,3)$ in (4) are

$$
\begin{aligned}
& C_{0}=\frac{1}{4} P_{1} \phi\left(A^{\dagger}\right) Q_{1}+\frac{1}{4} P_{2} \phi\left(A^{\dagger}\right) Q_{2} \\
& -\frac{1}{4} P_{3} \phi\left(A^{\dagger}\right) Q_{3}-\frac{1}{4} P_{4} \phi\left(A^{\dagger}\right) Q_{4} \\
& +\frac{1}{4}\left[W_{1}, W_{2},-W_{3},-W_{4}\right]\left[\begin{array}{c}
R_{\phi(A)} Q_{1} \\
R_{\phi(A)} Q_{2} \\
R_{\phi(A)} Q_{3} \\
R_{\phi(A)} Q_{4}
\end{array}\right], \\
& C_{1}=\frac{1}{4} P_{1} \phi\left(A^{\dagger}\right) Q_{2}-\frac{1}{4} P_{2} \phi\left(A^{\dagger}\right) Q_{1} \\
& +\frac{1}{4} P_{4} \phi\left(A^{\dagger}\right) Q_{3}-\frac{1}{4} P_{3} \phi\left(A^{\dagger}\right) Q_{4} \\
& +\frac{1}{4}\left[W_{1},-W_{2}, W_{4},-W_{3}\right]\left[\begin{array}{c}
R_{\phi(A)} Q_{2} \\
R_{\phi(A)} Q_{1} \\
R_{\phi(A)} Q_{3} \\
R_{\phi(A)} Q_{4}
\end{array}\right], \\
& C_{2}=\frac{1}{4} P_{1} \phi\left(A^{\dagger}\right) Q_{3}-\frac{1}{4} P_{2} \phi\left(A^{\dagger}\right) Q_{4} \\
& +\frac{1}{4} P_{3} \phi\left(A^{\dagger}\right) Q_{1}-\frac{1}{4} P_{4} \phi\left(A^{\dagger}\right) Q_{2} \\
& +\frac{1}{4}\left[W_{1}, W_{3},-W_{2},-W_{4}\right]\left[\begin{array}{c}
R_{\phi(A)} Q_{3} \\
R_{\phi(A)} Q_{1} \\
R_{\phi(A)} Q_{2} \\
R_{\phi(A)} Q_{4}
\end{array}\right], \\
& C_{3}=\frac{1}{4} P_{1} \phi\left(A^{\dagger}\right) Q_{4}+\frac{1}{4} P_{2} \phi\left(A^{\dagger}\right) Q_{3} \\
& +\frac{1}{4} P_{3} \phi\left(A^{\dagger}\right) Q_{2}+\frac{1}{4} P_{4} \phi\left(A^{\dagger}\right) Q_{1} \\
& +\frac{1}{4}\left[W_{1}, W_{4}, W_{3}, W_{2}\right]\left[\begin{array}{c}
R_{\phi(A)} Q_{4} \\
R_{\phi(A)} Q_{1} \\
R_{\phi(A)} Q_{2} \\
R_{\phi(A)} Q_{3}
\end{array}\right],
\end{aligned}
$$


where

$$
\begin{aligned}
& P_{1}=\left[I_{n}, 0,0,0\right], P_{2}=\left[0, I_{n}, 0,0\right], \\
& P_{3}=\left[0,0, I_{n}, 0\right], P_{4}=\left[0,0,0, I_{n}\right], \\
& Q_{1}=\left[I_{m}, 0,0,0\right]^{T}, Q_{2}=\left[0, I_{m}, 0,0\right]^{T}, \\
& Q_{3}=\left[0,0, I_{m}, 0\right]^{T}, Q_{4}=\left[0,0,0, I_{m}\right]^{T},
\end{aligned}
$$

and $W_{1}, W_{2}, W_{3}$ and $W_{4}$ are arbitrary real matrices with compatible sizes.

According to Lemma 2 and Theorem 3, we can get the following extreme ranks formulas for the real matrices in minimum norm g-inverse.

Theorem 2.8 Suppose that $A$ and $A^{(1,4)}$ are defined as (3) and (4). Then

a) $\max r\left(C_{0}\right)=\min \left\{r\left[\begin{array}{c}\overline{\bar{A}}_{0} \\ \sim \\ \tilde{A}\end{array}\right]-4 r(A)+m, 4 n\right\}$;

$$
\min r\left(C_{0}\right)=r\left[\begin{array}{c}
\overline{\bar{A}_{0}} \\
\widetilde{\widetilde{A}}
\end{array}\right]-r(\widetilde{\widetilde{A}}) \text {. }
$$

b) $\max r\left(C_{1}\right)=\min \left\{r\left[\begin{array}{c}\overline{\overline{A_{1}}} \\ \widetilde{\widetilde{A}}\end{array}\right]-4 r(A)+m, 4 n\right\}$;

$$
\min r\left(C_{1}\right)=r\left[\begin{array}{c}
\overline{\bar{A}}_{1} \\
\widetilde{\widetilde{A}}
\end{array}\right]-r(\widetilde{\widetilde{A}}) \text {. }
$$

c) $\max r\left(C_{2}\right)=\min \left\{r\left[\begin{array}{c}\overline{\bar{A}_{2}} \\ \widetilde{\widetilde{A}}\end{array}\right]-4 r(A)+m, 4 n\right\}$;

$$
\min r\left(C_{2}\right)=r\left[\begin{array}{c}
\overline{\bar{A}}_{2} \\
\widetilde{\widetilde{A}}
\end{array}\right]-r(\widetilde{\widetilde{A}}) \text {. }
$$

d) $\max r\left(C_{3}\right)=\min \left\{r\left[\begin{array}{c}\overline{\bar{A}}_{3} \\ \widetilde{\widetilde{A}}\end{array}\right]-4 r(A)+m, 4 n\right\}$;

$$
\min r\left(C_{3}\right)=r\left[\begin{array}{c}
\overline{\bar{A}}_{3} \\
\widetilde{\widetilde{A}}
\end{array}\right]-r(\widetilde{\widetilde{A}}),
$$

where

$$
\begin{aligned}
& \overline{\bar{A}}_{0}=\left[\begin{array}{llll}
A_{0} & -A_{1} & -A_{2} & -A_{3}
\end{array}\right], \\
& \overline{\bar{A}}_{1}=\left[\begin{array}{llll}
A_{1} & A_{3} & A_{3} & -A_{2}
\end{array}\right], \\
& \overline{\bar{A}}_{2}=\left[\begin{array}{llll}
A_{2} & -A_{3} & A_{0} & A_{1}
\end{array}\right], \\
& \overline{\bar{A}}_{3}=\left[\begin{array}{llll}
A_{3} & A_{2} & -A_{1} & A_{0}
\end{array}\right],
\end{aligned}
$$

$$
\begin{aligned}
& \widetilde{\widetilde{A}}=\left[\begin{array}{cccc}
\widetilde{\widetilde{A}}_{0} & 0 & 0 & 0 \\
0 & \widetilde{\widetilde{A}}_{1} & 0 & 0 \\
0 & 0 & \widetilde{\widetilde{A}}_{2} & 0 \\
0 & 0 & 0 & \widetilde{\widetilde{A}}_{3}
\end{array}\right], \\
& \widetilde{\widetilde{A}}_{0}=\left[\begin{array}{cccc}
-A_{2} & A_{3} & A_{0} & -A_{1} \\
A_{1} & A_{0} & -A_{3} & -A_{2} \\
A_{0} & -A_{1} & A_{2} & -A_{3}
\end{array}\right] \text {, } \\
& \widetilde{\widetilde{A}}_{1}=\left[\begin{array}{cccc}
A_{3} & A_{2} & A_{1} & A_{0} \\
A_{1} & A_{0} & -A_{3} & -A_{2} \\
A_{0} & -A_{1} & A_{2} & -A_{3}
\end{array}\right] \text {, } \\
& \widetilde{\widetilde{A}}_{2}=\left[\begin{array}{cccc}
A_{3} & A_{2} & A_{1} & A_{0} \\
-A_{2} & A_{3} & A_{0} & -A_{1} \\
A_{0} & -A_{1} & A_{2} & -A_{3}
\end{array}\right] \text {, } \\
& \widetilde{\widetilde{A}}_{2}=\left[\begin{array}{cccc}
A_{3} & A_{2} & A_{1} & A_{0} \\
-A_{2} & A_{3} & A_{0} & -A_{1} \\
A_{1} & A_{0} & -A_{3} & -A_{2}
\end{array}\right] .
\end{aligned}
$$

As one of important applications of the maximal and minimal ranks to real matrices, Theorem 4 can help to get the necessary and sufficient conditions for the existence of some special minimum norm g-inverse. We show them in the following.

Corollary 2.9 Suppose $A=A_{0}+A_{1} i+A_{2} j+A_{3} k$. Then

a) A quaternion matrix $A$ has a real minimum norm $g$-inverse if and only if

$$
r\left[\begin{array}{c}
\overline{\bar{A}}_{1} \\
\widetilde{\widetilde{A}}
\end{array}\right]=r\left[\begin{array}{c}
\overline{\bar{A}}_{2} \\
\widetilde{\widetilde{A}}
\end{array}\right]=r\left[\begin{array}{c}
\overline{\bar{A}}_{3} \\
\widetilde{\widetilde{A}}
\end{array}\right]=r(\widetilde{\widetilde{A}}) .
$$

b) All minimum norm g-inverse of quaternion matrix $A$ are real matrices if and only if

$$
r\left[\begin{array}{c}
\overline{\bar{A}}_{1} \\
\widetilde{\widetilde{A}}
\end{array}\right]=r\left[\begin{array}{c}
\overline{\bar{A}}_{2} \\
\widetilde{\widetilde{A}}
\end{array}\right]=r\left[\begin{array}{c}
\overline{\bar{A}}_{3} \\
\widetilde{\widetilde{A}}
\end{array}\right]=4 r(A)-m,
$$

where $\overline{\bar{A}}_{1}, \overline{\bar{A}}_{2}, \overline{\bar{A}}_{3}$ and $\widetilde{\widetilde{A}}$ are defined as Theorem 4 .

Corollary 2.10 Suppose $A=A_{0}+A_{1} i+A_{2} j+A_{3} k$. Then

a) A quaternion matrix $A$ has a pure imaginary minimum norm $g$-inverse if and only if

$$
r\left[\begin{array}{c}
\overline{\bar{A}}_{0} \\
\widetilde{\widetilde{A}}
\end{array}\right]=r(\widetilde{\widetilde{A}}) \text {. }
$$

b) All minimum norm g-inverse of quaternion matrix $A$ are pure imaginary matrices if and only if

$$
r\left[\begin{array}{c}
\overline{\bar{A}}_{0} \\
\widetilde{\widetilde{A}}
\end{array}\right]=4 r(A)-m,
$$

where $\overline{\bar{A}}_{0}$ and $\widetilde{\widetilde{A}}$ are defined as Theorem 4 . 


\section{REFERENCES}

[1] T. W. Hungerford, “Algebra,” Spring-Verlag Inc., New York, 1980.

[2] A. Ben-Israel and T. N. E. Greville, "Generalized Inverses: Theory and Applications,” R. E. Krieger Pubilshing Company, New York, 1980.

[3] H. Yanai, "Some Generalized Forms a Least Squares g-Inverse, Minimum Norm g-Inverse, and Moore-Penrose Inverse Matrices,” Computational Statistics \& Data Analysis, Vol. 10, No. 3, 1990, pp. 251-260. doi:10.1016/0167-9473(90)90005-3

[4] Y. Tian, "More on Maximal and Minimal Ranks of Schur Complements with Applications," Applied Mathematics and Computation, Vol. 152, No. 3, 2004, pp. 675-692. doi:10.1016/S0096-3003(03)00585-X

[5] Y. Tian, "Schur Complements and Banachiewicz-Schur Forms,” Electronic Journal of Linear Algebra Society, Vol. 13, 2005, pp. 405-418.

[6] W. Guo, M. Wei and M. Wang, “On Least Squares g-Inverses and Minimum Norm g-Inverses of a Bordered Matrix,” Linear Algebra and its Applications, Vol. 416, No. 2-3, 2006, pp. 627-642. doi:10.1016/j.laa.2005.12.015

[7] S. L. Adler, "Quaternionic Quantum Mechanics and Quantum Fields,” Oxford University Press, Oxford, 1995.

[8] F. Zhang, "Quaternions and Matrices of Quaternions," Linear Algebra and its Applications, Vol. 251, No. 15, 1997, pp. 21-57. doi:10.1016/0024-3795(95)00543-9

[9] C. E. Moxey, S. J. Sangwine and T. A. Ell, "Hypercomplex Correlation Techniques for Vector Images,” IEEE Transactions on Signal Processing, Vol. 51, No. 7, 2003, pp. 1941-1953. doi:10.1109/TSP.2003.812734
[10] N. LE Bihan and J. Mars, "Singular Value Decomposition of Matrices of Quaternions: A New Tool for Vector-Sensor Signal Processing,” Signal Processing, Vol. 84, No. 7, 2004, pp. 1177-1199. doi:10.1016/j.sigpro.2004.04.001

[11] N. Le Bihan and S. J. Sangwine, "Quaternion Principal Component Analysis of Color Images,” IEEE International Conference on Image Processing (ICIP), Barcelona, 14-17 September 2003, pp. 809-812.

[12] N. Le Bihan and S. J. Sangwine, "Color Image Decomposition Using Quaternion Singular Value Decomposition,” IEEE International conference on Visual Information Engineering (VIE), Guildford, 7-9 July 2003, pp. 113-116.

[13] S. J. Sangwine and N. Le Bihan, "Quaternion Singular Value Decomposition Based on Bidiagonalization to a Real or Complex Matrix Using Quaternion Householder Transformations," Applied Mathematics and Computation, Vol. 182, No. 1, 2006, pp. 727-738. doi:10.1016/j.amc.2006.04.032

[14] Q. W. Wang, H. S. Zhang and S. W. Yu, "On Solutions to the Quaternion Matrix Equation $A X B+C Y D=E$," Electronic Journal of Linear Algebra, Vol. 17, 2008, pp. 343358.

[15] S. K. Mitra, "A Pair of Simultaneous Linear Matrix Equations and a Matrix Programming Problem," Linear Algebra and Its Applications, Vol. 131, No. 1, 1990, pp. 97-123. doi:10.1016/0024-3795(90)90377-O

[16] Y. Tian, "The Maximal and Minimal Ranks of Some Expressions of Generalized Inverses of Matrices," Southeast Asian Bulletin Mathematics, Vol. 25, No. 4, 2002, pp. 745-755. doi:10.1007/s100120200015 